\title{
Improving Nursing Documentation via Reducing the Written Work Load
}

\author{
Anaiz Ahmed ${ }^{1 *}$ and Nasreen Rafiq ${ }^{2}$ \\ ${ }^{1}$ MSc in health policy and management, Head Nurse at Coronary Care Unit, Aga Khan University, Pakistan \\ ${ }^{2}$ Registered Nurse at Intensive Care unit, Aga Khan University Hospital
}

*Corresponding author: Anaiz Ahmed, Head Nurse, Coronary Care Unit, Aga Khan University, Pakistan.
Received Date: January 16, 2019

Published Date: February 19, 2019

\begin{abstract}
With the growth in the health care industry around the world and introduction to new technologies in the system, the uses of traditional methods in nursing practices are reducing. These modern work practices are meant to reduce human effort without compromising on the quality. Where as dealing with documentation whereas always has been a major challenge in providing the better health care. The project thus outlines the improving nursing documentation via reducing the written work load to increase work efficiency.

Abbreviations: HN: Head Nurse; CTS: Cardio Thoracic Surgery; RN: Register Nurse; HCA: Health Care Assistance; QMHS: Quality Management in Health Services; JCIA: Joint Omission International Accreditation; PDSA: Plan-Do-Study-Act; CQI: Continuous Quality Improvement; LAMA: Leave Against Medical Advice; CEO: Chief Executive Officer
\end{abstract}

\section{Introduction}

In this world, the healthcare has become increasingly demanding. The complex environment of the healthcare system and high customer demands creates challenging task for the healthcare professionals. In this situation hospitals endeavor to contemplate on patient care and increased patient altitude of satisfaction. One of the major vicinities of patient care is "nursing care" where nurses' available 24 hours to full fill patient requirements. With this belief, nursing profession has gain more responsibility as they are the frontline of healthcare delivery. They are linked with direct delivering of patient centered care and high level of outcomes and patient satisfaction. However, the turn over rate has increased for varous factors which lead to nursing turnover rate and effect patient care.

\section{Back Ground and Literature Review}

The availability of nursing staff is associated with positive patient outcomes in clinical settings [1]. In the same context to improve the quality of patient care, Major hospital administrators are fascinated in increasing the amount of time nurses have available for direct patient care by reducing the amount of time nurses spend in written documentation. As nursing documentation is important aspect but Instead, proficiency improvements are required, that documentation of corresponding quality and precision can be inputted in less time as a result more time to be spent on direct patient care.

A study conducting with sample size of 2160 nurses in 105 units and 55 hospitals shows that day-shift nurses spent $19.2 \%$ of shifts documenting. In the study it is mentioned that the different categories used and associated percentage of time shows that nurses give $(47 \%)$ of their time to direct care of patient, $(19 \%)$ of their shift time on documentation, moreover they involve in indirect care (18\%), (5\%) in administration work, (5\%) in personal work, (3\%) of the time they waste and remaining (3\%) in other activities [2]. This study shows that nurses utilized major junction of their times on documentation rather than involvement in direct nursing care. Another study conducting by Niklas et al. showed that $45.8 \%$ of time by nurses was spent on patient care, $16.5 \%$ on indirect patient care, $25.6 \%$ they utilize their time on documentation and $12.0 \%$ on miscellaneous tasks. From all these studies it is concluded that as nurses utilize most of their time in nursing documentation which should be minimize by improving training, removing unnecessarily documentation and encourage use of time saving tools. 


\section{Problem Statement}

Reducing the nursing written work load burden while implementing NCP plans in to the admission forms.

\section{Rational}

1. Years of research show that safer nurse staffing levels directly improve patient safety, health outcomes and quality of care, yet in many countries around the world, nurses continue to experience excessive workloads and patients and families suffer as a result.

2. The Goal of the hospital for Feb 2016 is to reduce nursing work load by decreasing nursing documentation and making it effective, which in long run would reduce the turnover of the nurses.

\section{Project goal}

The project goal is continuous patient care quality improvement in a Cardio thoracic ward of a tertiary care hospital.

\section{Objective}

a. To identify gaps in quality patient care leading to patient dissatisfaction in D1 Ward of a tertiary care hospital.

b. To review the methods and processes employed for quality patient care to identify factors contributing to patient dissatisfaction in D1 Ward of a tertiary care hospital.

c. To suggest possible strategies to overcome Nursing documentation issue in D1 of a tertiary care hospital.

\section{Methodology}

\section{Study setting}

\section{Data collection tools}

A checklist was developed and pre-tested from May 01st till June 01st 2016. The tool was developed and approved from department manager and project facilitator. The tool was also sent to three bio-statisticians of the Aga Khan University Hospital for ensuring its validity and reliability, and the average of their content validity index (CVI) results was 0.85 .

\section{Sampling}

The number was obtained by calculating the mean difference by the total number of nurses and self-designed inclusion and exclusion criteria.

\section{Data collection methods}

1. Active observation of RNs from D1 ward.

\section{Data collection duration}

Data was collected between May 01st till June 01st 2016.

\section{Situation Analysis}

It comprises of total 53 beds which include 1 pulmonology special cares, 1 cardiac step down, 2 cardiothoracic step down, 3 blocks of general ward and 8 rooms of semi private.

\section{In 24 hours, total average of admission and discharges}

1. 15 new admissions including from ER and electively for surgery.

2. 8 transfer in patients from different of service line 1 .

3. 18 discharges from the floor including LAMA .

4. 8 transfer out (alive) patients either to others ward or outside other hospitals.

\section{In 24 hours totals RNs and HCAs}

1. There are total three shifts each including 8 RNs, 7 nursing Assistance per shifts.

2. Critical area nurse patient ratio is $1: 5$ and in general ward area 1:10 (Figure 1\&2).

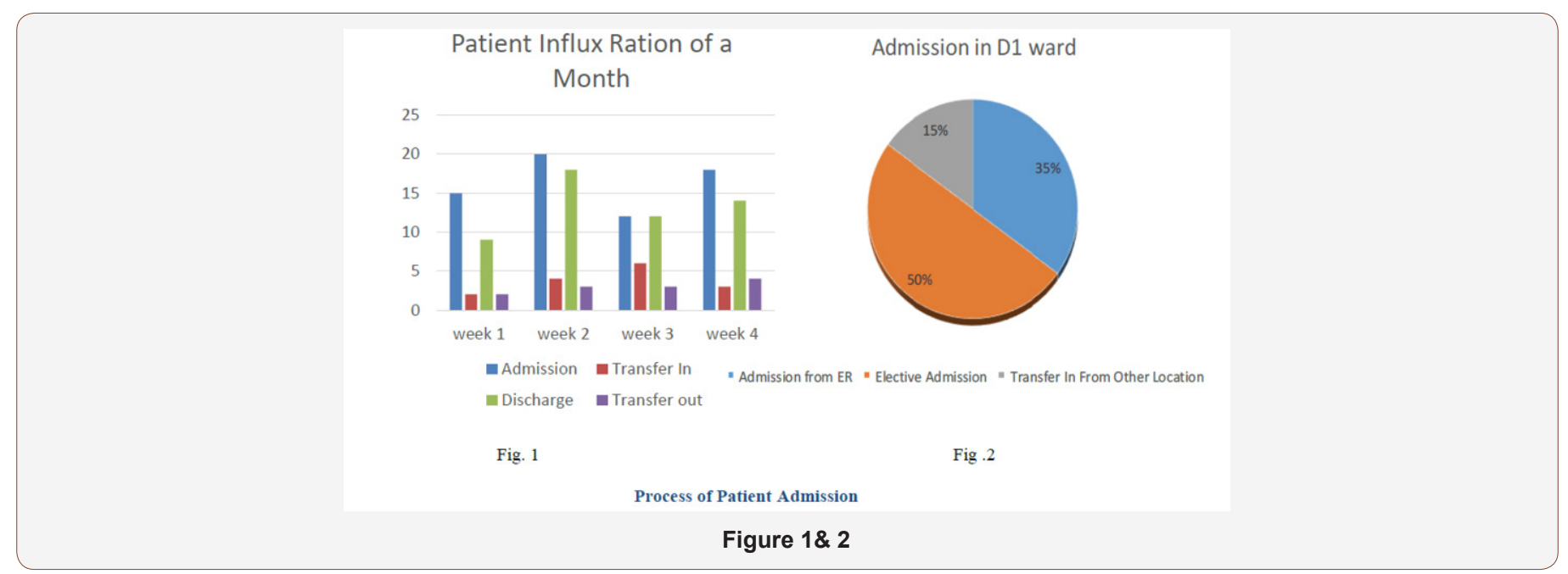

\section{Methodology}

\section{Inputs}

As patient admitted to D1 ward either from ER or electively admitted for any procedure or shifted from other ward.

\section{Process}

As patient admitted register nurses initially perform height and weight and guide the patient on assign bed. After that, nurses attain initial vital signs and receiving notes in bed side folder, do IV cannulation and send required labs as order by doctor. 
Meanwhile Unit receptionist inform the resident of respected team. Subsequently, assigned nurse fill the initial assessment form which almost take 25 to 30 minutes. Apart from the problem list, all the columns in the initial assessment form is designed in the form of check boxes, which makes the life of a nurse much easier. However, the problem list is in fact the nurse's diagnosis, which a nurse has to identify after acquiring the all data from the patient.

\section{Output}

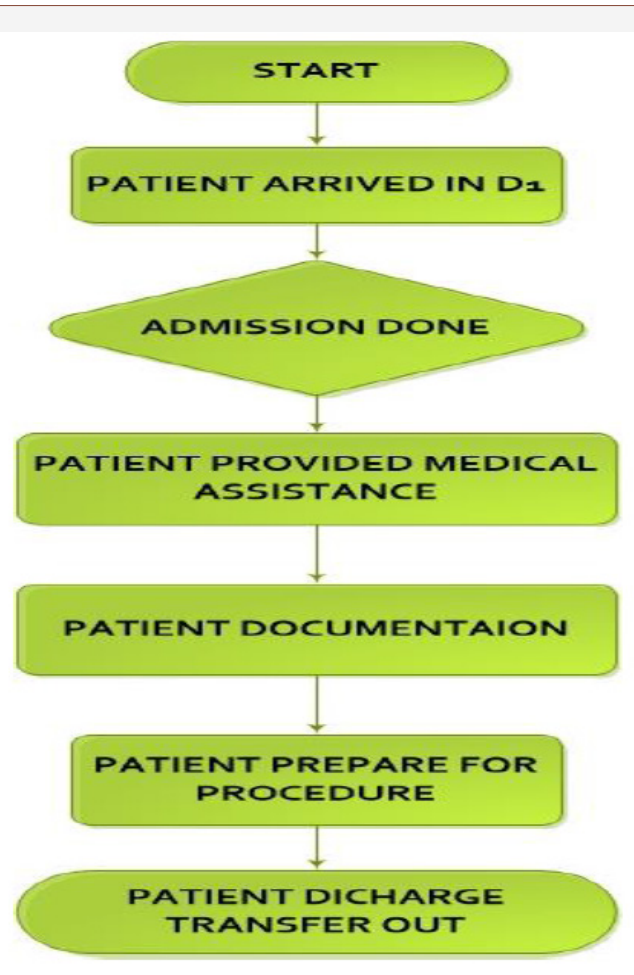

Figure 3

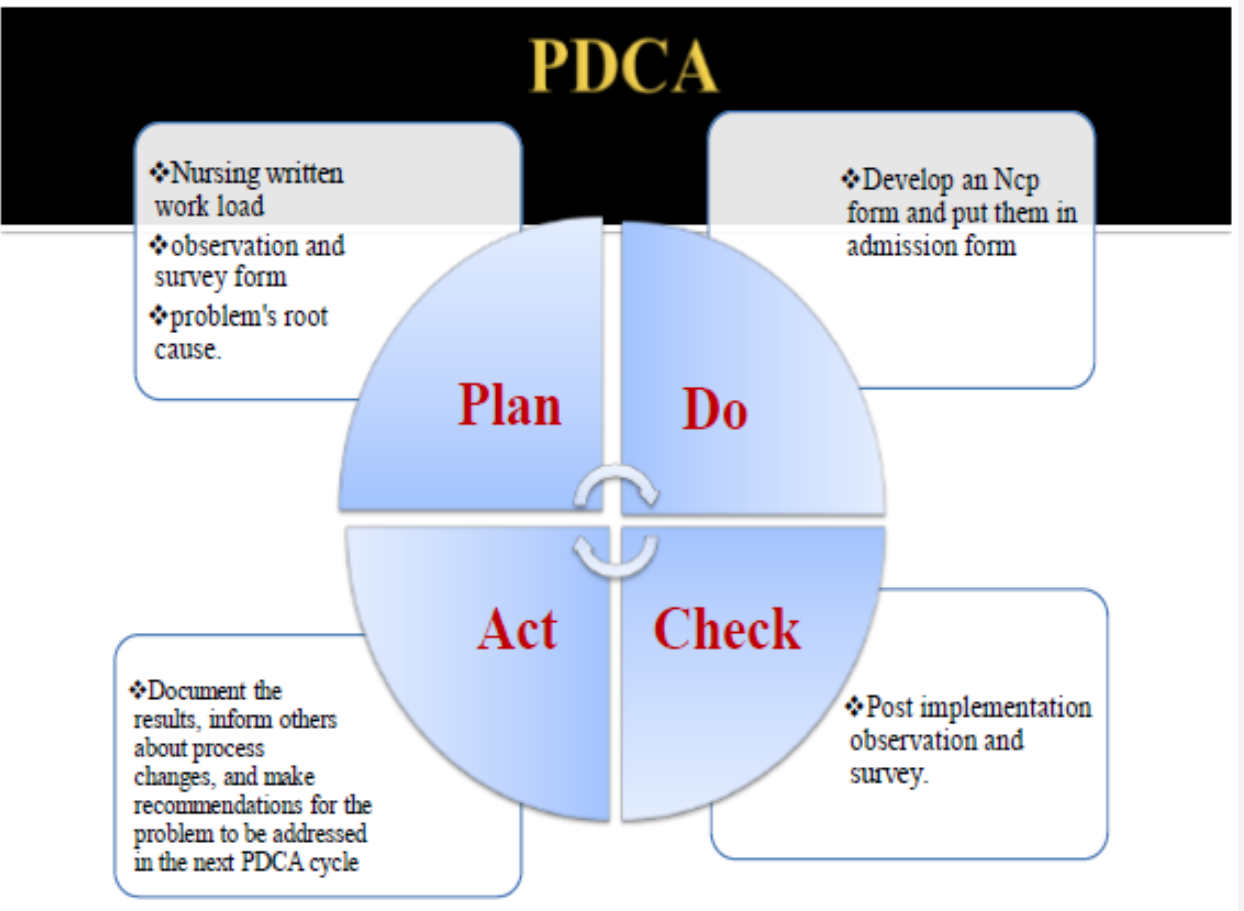

PDCA-CYCLE FOR REDUCING NURSING DOCUMENTION WORK LOAD

Figure 4 
To further simplify the process and save nurses time, we made an NCP index as shown in fig 5 and put them in initial assessment in which they have to simply check the relevant box or column and add their related problems. We predicted that after applying posttest, it would assist nurses to almost safe 10 to 15 minutes for every new patient they receive, and it would also assist nurses to care for patient pertinent to the nursing diagnosis which are relevant to the patient's problem. As this is an academic project due to time limitation, we limited to the plane phase till now and will implement in our selected in near future with the permission and support of our nusing manger, clinical nurse supervisor and head nurse. By our experienced, observations, litures review and evidence we get and analyses the data and find the quality gaps and suggest recommendation through PDSA cycle/Shewart cycle in (Figure $3 \& 4$ ) below. Initially by our observations, experience and different literature reviews and studies we find that through Improving Nursing Documentation via Reducing the Written Work Load as a result to enhance patient satisfaction by providing quality care to them.

\section{Pareto analysis}

We identified different factors responsible for nursing turnover rate in which low salary, increase documentation and respect and recognition to nurses lead to $80 \%$ of because which leads to nursing turnover as result patient quality care compromises. As in the hospital, the higher management is working on low salaries here we pick the second major reason increase nursing documentation workload as the problem of concern to reduce it. We analyzed this data using Pareto chart and categorized the factors causing increased nursing turnover.

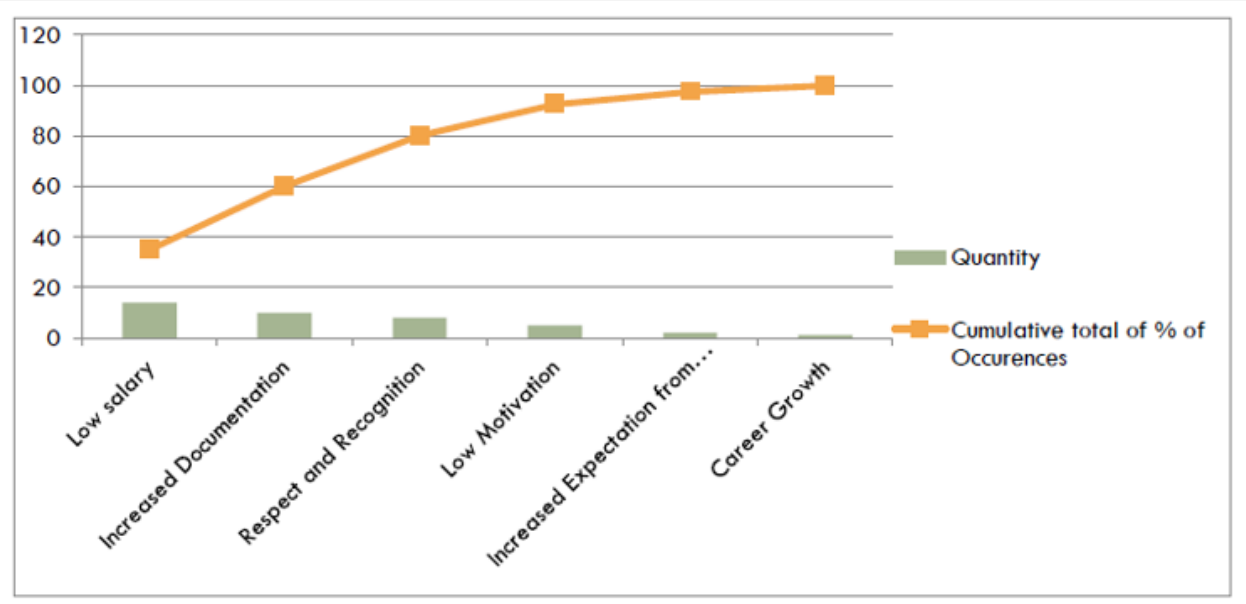

Figure 5

\section{Cause and effect diagram}

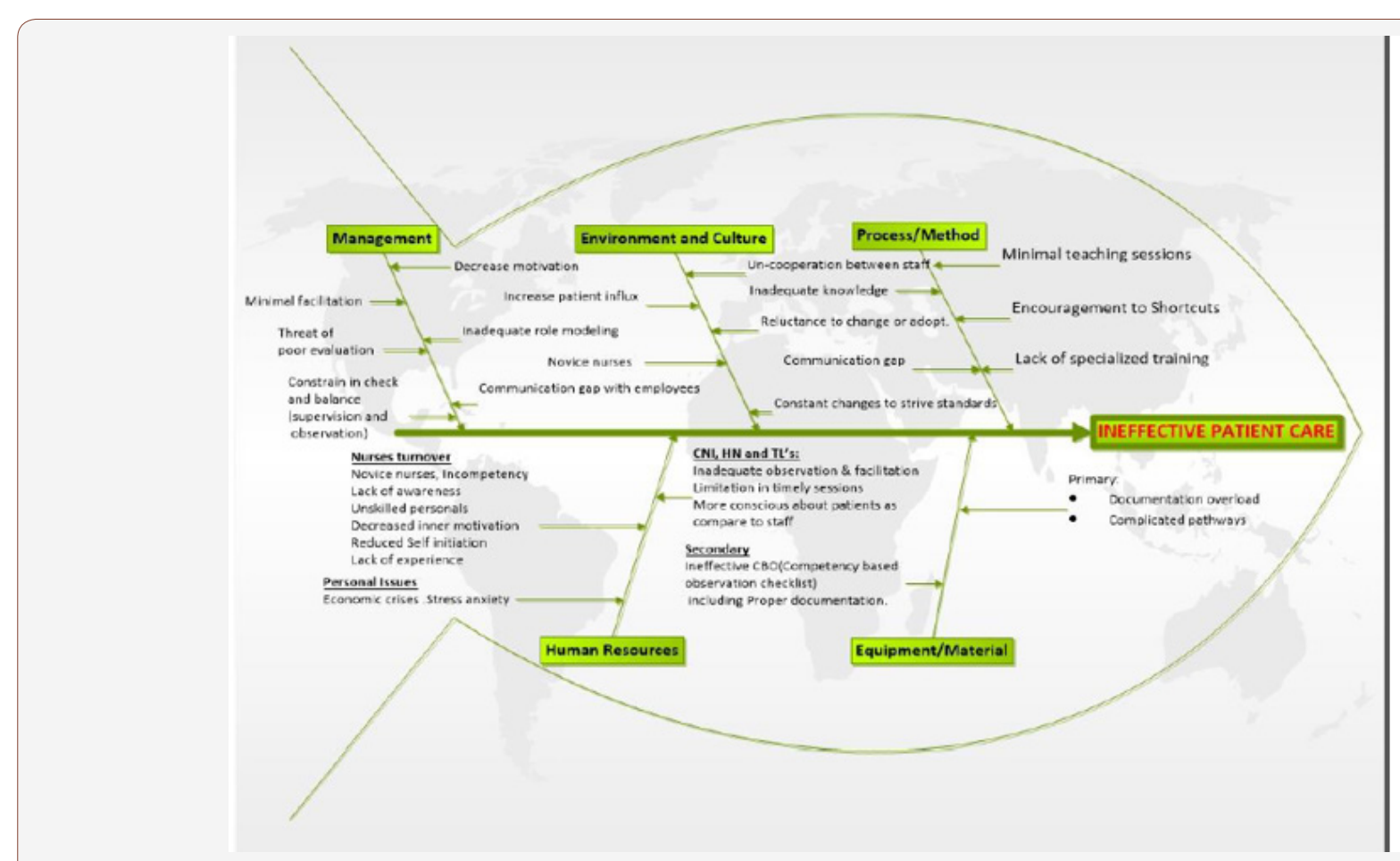

Figure 6 
Through fish bone diagram we found different causes of ineffective patient care by observation and collected data from 20 RNs via self made checklist and a survey form and find root cause analysis and described in cause \& effect diagram shown below in figure. We divided the identified factors into five major domains. These are Management, environment and culture, process/ methods, human resources and equipment/materials.

\section{Do Phase}

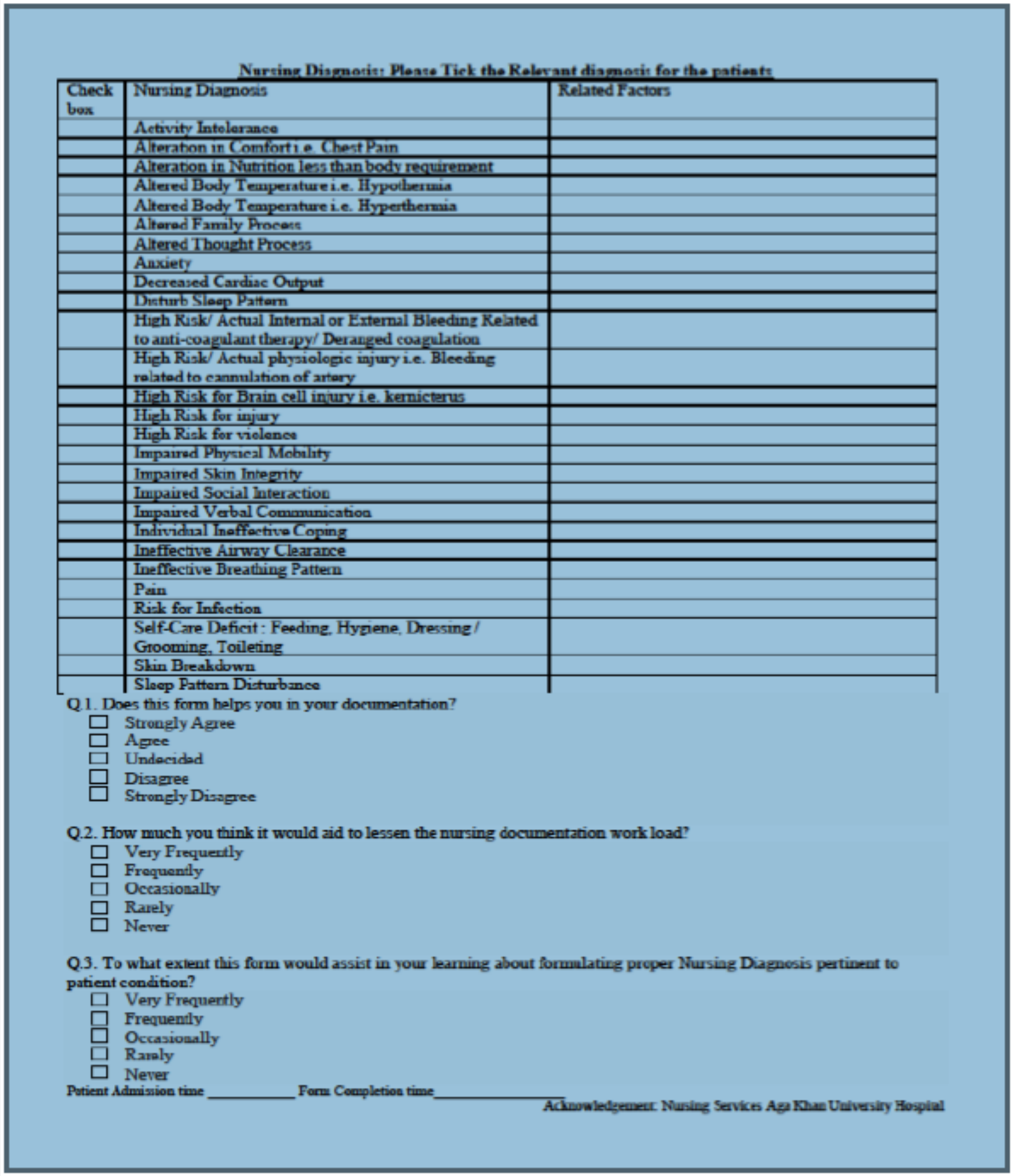

Figure 7

As we found that nurses were spending average 20 minutes on nursing diagnosis in initial form, so we made a simple checklist and put them in initial assessment form as pilot project. It is easy for them to check the box and write their secondary related problem in order to save their time and prevent any errors. We also tried to find the perception of nurses through 3 Likert scale question, identifying nurses idea about changing and modifying nurses initial assessment form. 


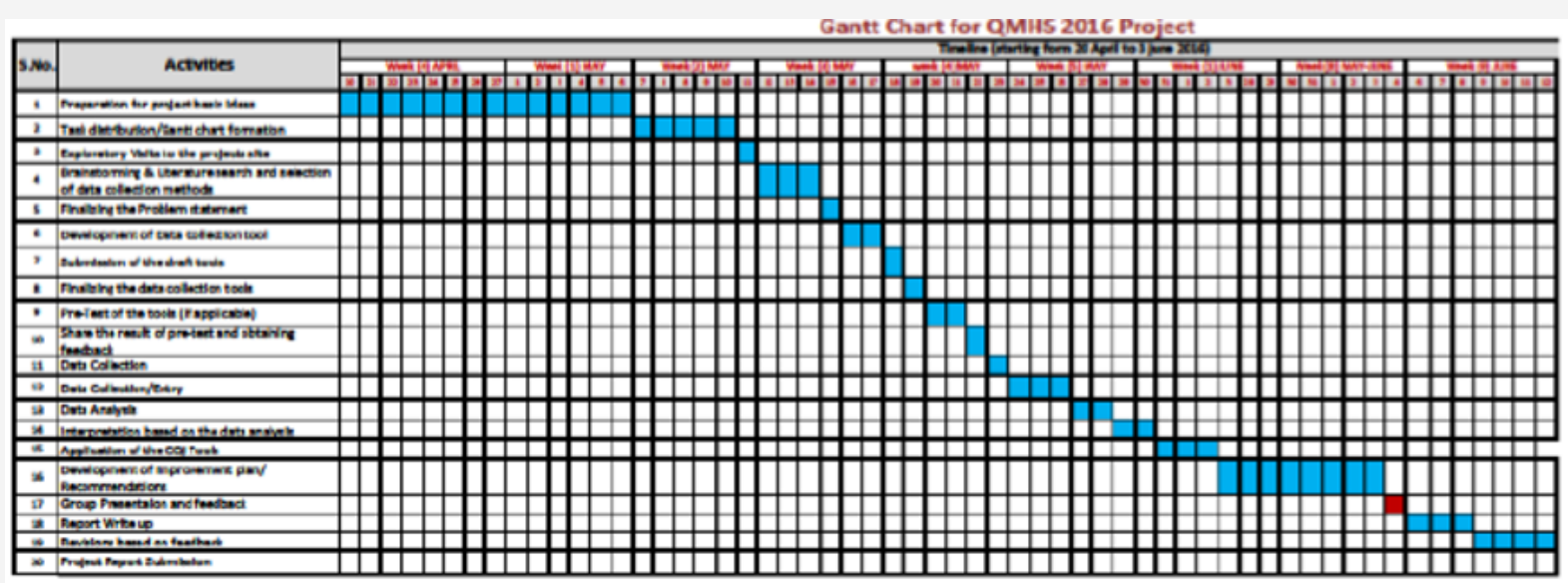

Figure 8

\section{Study phase}

In this phase we get the result and analysis via Run Chart Matrix and it was found that Data analysis is done by comparing pre and post intervention. Data Analysis is done by using bar Graph shown below.

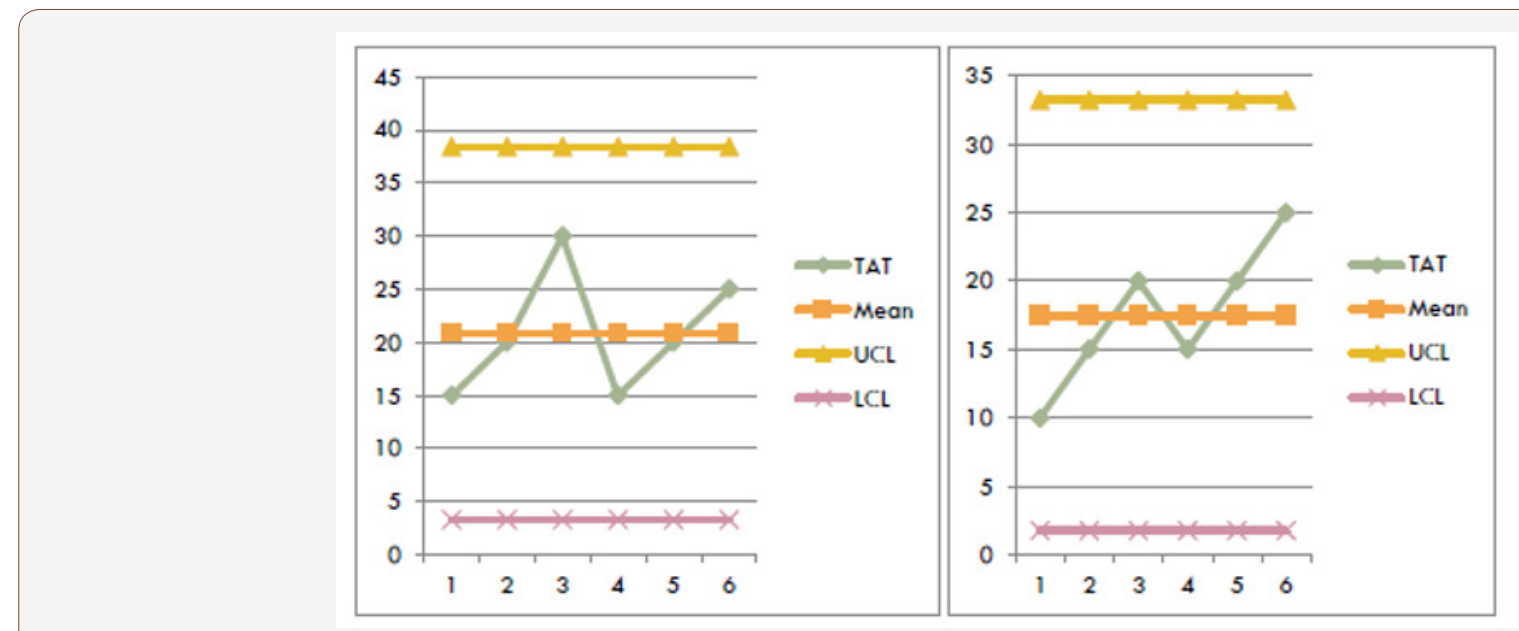

Figure 9

\section{Act phase}

So, with the study about this pilot project, we have planned to replicate this study design in to other areas to see the wider change difference in the hospital. We highly belief that this project will serve as the basic for the other several project for reduce burden on nurses in inpatient area.

\section{Results}

If was found that earlier to implementation the mean time for nurses to complete the documentation was 22 min average which range from 30 to $15 \mathrm{~min}$, depending on the competences and skills of a nurse. However, it had a vast difference which was causing increased time spending in to documentation and also resulting in error due to lack of timings. Furthermore, it was observed that after implementing the new form the mean got shifted to around 18 mins achieving good results from ranging 10 to $25 \mathrm{~min}$ on average. It also enhanced the knowledge of the novice nurses and provided them an opportunity to get empowered about taking care of their patient's particular to their requirement. It can also be inferred that after the initial phase, once nurses would get familiarize with this format, it would further aid in achieving reducing time spending in documentation.

\section{Future Recommendation}

1. Active \& responsive leadership.

2. Putting NCP index in Initial Assessment.

3. Supervision of trainees to assure quality control.

4. Quarterly review of operational values.

5. Capacity building of Nurses.

6. Electronic documentation.

\section{Ethical Consideration}

The study ensured the anonymity of the participants. A written permission was obtained from the head of the department and then data collection was started. The study also ensures the principles of autonomy and confidentiality.

\section{Conclusion}

To conclude with this intervention and project work, it is very significant to work in the direction of making more convenient form of documentation for nurses. Because nurse's prime responsibility is to take of the patient by actually getting involved in patient care. 
However, documentation is also of prime importance therefore we cannot neglect that part. But yes, we can always come up with the new modified and simplified forms of documentation to aid saving nurse's time. Since past we are working in this direction and it has achieved far better results and continuing this process can more help to reduce nurse's burden.

\section{References}

1. Needleman J, Buerhaus P, Mattke S, Stewart M, Zelevinsky K (2002) Nurse-staffing levels and the quality of care in hospitals. The New England Journal of Medicine 346(22): 1716-1722.
2. Korst LM, Eusebio Angeja AC, Chamorro T, Aydin CE, Gregory KD (2003). Nursing documentation time during implementation of an electronic medical record. JONA: The Journal of Nursing Administration 33 (1): 24-30.

3. Yee T, Needleman J, Pearson M, Parkerton P, Parkerton M, Wolstein J (2012) The influence of integrated electronic medical records and computerized nursing notes on nurses' time spent in documentation. CIN: Computers Informatics Nursing 30(6): 287-292.

4. Hamid S, Malik A, Kamran I, Ramzan M (2014) Job satisfaction among nurses working in the private and public sectors: a qualitative study in tertiary care hospitals in Pakistan. J Multidiscip Healthc 7(3): 25-35. 